\title{
Vibrations reduction in milling by using singular spectral analysis
}

\author{
Vladimir Kuts ${ }^{1, *}$, Igor Kiselev ${ }^{1}$, and Sergey Voronov ${ }^{1}$ \\ ${ }^{1}$ Bauman Moscow State Technical University, 5/1, Ul. Baumanskaya 2-ya, Moscow 105005, Russia
}

\begin{abstract}
The main issue which limits the milling process efficiency is the emergence of the self-excited vibrations ("chatter") during milling. The system "tool-workpiece" autooscillations lead to more rapid tool and machine units deterioration, the decrease in quality of the machined surface. The methodology of the self-excited vibrations detection during milling, which allows to choose the most favorable and efficient modes (free of "chatter"), is represented in this article. This methodology is based on the singular spectral analysis of the system acceleration signals. The methodology is tested out on the example of flat milling. The system "toolworkpiece" accelerations signals were received using the special-purpose software 3DCUT. The "map of modes" was drawn and the most favorable mode of milling were chosen as a result of the analysis of the system accelerations signals.
\end{abstract}

\section{Introduction}

The main issue which limits the milling process efficiency is the unwanted vibrations [1]. The particular feature of the milling is the intermittent cutting process, which is why the vibrations always emerge in the system «tool/workpiece». The mechanisms of vibrations which emerge during milling are quite different - from forced to self-excited vibrations[24]. The forced vibrations of the system emerge due to the intermittent nature of the cutting forces. The displacements and cutting forces amplitudes are not high in this case and it has a positive impact on the process quality. The certain combination of thechnological parameters may cause the self-excited vibrations emergence in the system « tool/workpiece » due to the regenerative mechanism. The consequences of this effect are a multiple increase in the cutting forces, vibrations amplitudes, more rapid tool and machine units deterioration, and also the decrease in quality of the machined surface $[3,4]$. The issue of the self-excited vibrations is especially of high relevance during the compliant parts machining, which are made out of the hard-to-machine materials, for example, gasturbine engines blades and blings. Thus, to ensure the required quality of the machined surface and the increase in the milling process efficiency, it is needed to thoroughly choose the machining modes parameters and avoid the modes, in which the self-excited vibrations emerge.

\footnotetext{
*Corresponding author: vlankuts@gmail.com
} 
Several srtategies have been suggested till now, the aim of which is the prediction and suppression of the self-excited vibrations during milling: the analysis of stability of the milling dynamics equations; the modelling of the milling dynamics with the usage of the finite elements method; the self-excited vibrations detection using signals, which are being measured during machining; the modification of the system «spindle/tool/workpiece » parts.

The most significant results were obtained using the methods which imply the analysis of stability of the milling dynamics equations in pieces of research [5-7]. The mathematical models, which describe the machining process dynamics, were developed in these articles, the mechanism of the surface regeneration was described and the methods of analysis and prediction of the self-ecxited vibrations were suggested. The result of the stability analysis is the stability lobes diagram (figure 1).

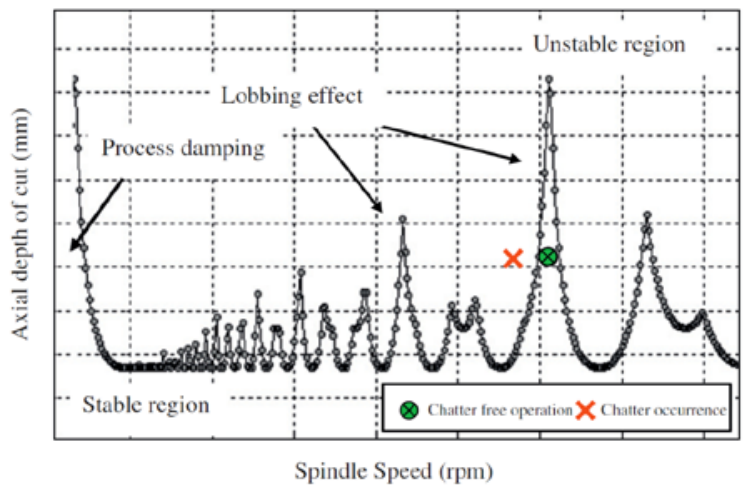

Fig. 1. The stability lobes diagram.

The stability lobes diagram allows to determine whether or not the vibrations level will increase during milling with the defined parameters. It is needed to add that the workpiece dynamics has not been taken into consideration in the majority of researches using this methodology. That is why, this methodology can not be used if the workpiece is compliant.

The finite elements method has been commonly used recently to predict vibrations. The methods using such kind of approach allow to model the system «tool/workpiece » behaviour during machining taking into account its dynamics. The results of modeling are the vibrations, cutting forces, instantaneous cut-off thickness amplitudes and the geometry of the machined surface $[8,9]$. The analysis of the modeling results allows to infer the selfexcited vibrations occurrence in the system and define the optimal machining parameters.

The self-excited vibrations detection is an alternative method to control the machining process and choose the optimal machining mode. This kind of approach is based on the analysis of the signal, which is being received in real-time mode during milling. As a result of this analysis, it will be decided whether "chatter » occurs in the system or not, and if it is needed, certain machining parameters are to be changed. The majority of the detection methods, which are put into practice, is based on the representation of the signal in the frequency domain and its spectrum analysis [2] (the Fourier transform, wavelet transformation, filtering and demodulation). The high speed and quality requirements of the signal processing are to be met by such kind of methods. The detection happens to be quite labour-intensive due to the signal noise pollution and the process complexity.

The self-excited vibrations detection method using the singular spectral analysis and its test-out on the flat milling process have been described briefly in this article. The developed methodology allows to predict the self-excited vibrations occurrence and define favorable and efficient machining modes. 


\section{The detection method description}

The experimental and analytical research into the milling dynamics showed that during the favorable machining modes there are much more harmonics with frequencies which are divisible by the frequency factor of the cutting edges $\left(\omega_{k}, k=1,2,3 \ldots n\right)$ in the acceleration and cutting forces signals spectrum. If the self-excited vibrations appear in the system, there are harmonics (in the signals spectrum) with frequencies («chatter») which are not divisible by the frequency factor of the cutting edges, but are near the system natural frequency. That is why, the analysis of the components, which form the monitored signal, allows to infer the process quality.

The self-excited vibrations detection methodology developed by the authors is based on the system «cutter/workpiece » accelerations signals, which are being registered during the machining process. The signal processing algorithm is based on the singular spectrum analysis (SSA) [10]. The usage of the SSA allows to decompose the registered signal into the separate components, which are harmonics and noise in case of the milling. The identification of the harmonics parameters (the frequency and amplitude) allows to infer the « chatter » frequency occurence and its contribution to the signal amplitude.

\subsection{Decomposing the signal into components}

Let us consider the time series $f(\mathrm{t})$, which is formed by the sequence of the equidistant values of certain function :

$$
f=\left[f_{0}, f_{1}, f_{2} \ldots f_{N-1}\right]
$$

The trajectory Hankel matrix (1) is being formed based on the time series :

$$
X=\left[\begin{array}{ccccc}
f_{0} & f_{1} & f_{2} & \ldots & f_{k-1} \\
f_{1} & f_{2} & f_{3} & \ldots & f_{k} \\
f_{2} & f_{3} & f_{4} & \ldots & f_{k+1} \\
\vdots & \vdots & \vdots & \ddots & \vdots \\
f_{l-1} & f_{l} & f_{l+1} & \ldots & f_{N-1}
\end{array}\right] \in R^{l \times k}
$$

The parameter $l$, which equals to the number of the rows of the trajectory matrix, is to be chosen divisible by the period of the harmonic with the frequency factor of the cutting edges [10]. The parameter $k$ is to be calculated using the formula (2).

$$
k=N-l+1
$$

The next step is the singular value decomposition (SVD) of the singular matrix $X(3)$.

$$
X=U \Lambda V^{T}
$$

where $\Lambda \in R^{l \times k}$ - the diagonal matrix of the singular values of the matrix $X$ in a decreasing order, $U \in R^{l \times l}$ и $V \in R^{k \times k}$ - the unitary matrices of the left-singular and the right-singular vectors correspondingly.

Based on the values of the matrix of the singular values, the grouping of the components and the filtering of the time series are carried out. The trajectory matrix $X_{i}$ of the separate component of the series $f$ (4) is calculated as a result of the grouping and its component (5) is restored by the diagonal averaging. 


$$
\begin{gathered}
X_{i}=\sqrt{\lambda_{i}} u^{i}\left(\mathrm{v}^{i}\right)^{T} \\
g^{s}=\left\{\begin{array}{l}
\frac{1}{s+1} \sum_{m=1}^{s+1} y_{m, s-m+2}, \quad 0 \leq s \leq l-1 \\
\frac{1}{l} \sum_{m=1}^{l} y_{m, s-m+2}, \quad l-1 \leq s \leq k \\
\frac{1}{N-s} \sum_{m=s-k+2}^{N-k+1} y_{m, s-m+2}, \quad k \leq s \leq N
\end{array}\right.
\end{gathered}
$$

Thus, the vector $\boldsymbol{g}^{s}$ is the value of the i-th component of the time series $f$. More detailed description of the algorithm of the time series decomposition is represented in [10].

\subsection{The signal components analysis}

The usage of the SSA algorithm allows to analyze several signal components at the same time and independently. Furthermore, the usage of the singular value decomposition (SVD) (3) in this algorithm allows to extract components, which contribute the most to the signal amplitude, which simplifies the grouping. Based on the analysis of component's frequency and amplitude, which form a signal, the conclusion is drawn whether there is " chatter » in the system or not and the degree of danger. More detailed description of the " chatter " frequency detection stages is represented in the piece of research [11].

\section{The detection algorithm test-out on the example of milling}

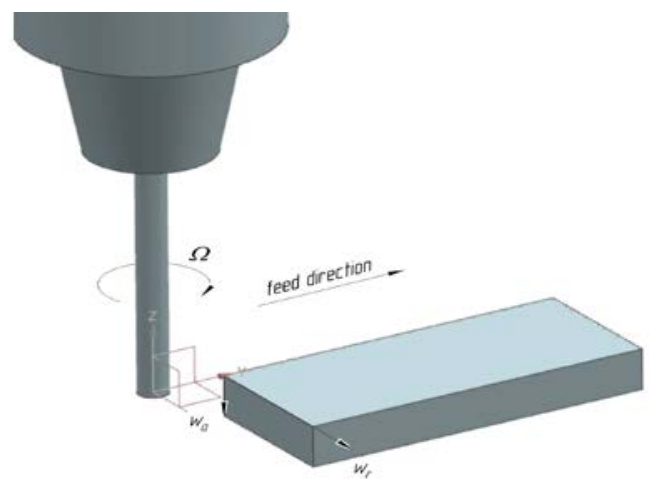

Fig. 2. The tool and workpiece models for the milling modeling.

The multivariant modeling of the milling dynamics was carried out in the software package 3DCUT [12] to receive the system accelerations signals. Each multivariant calculation was executed for different values of the spindle rotational speed and constant values of feed, axial and radial depth of cut.

The model of the tool and workpiece is represented in the figure 2.

The straight cutter, the parameters of which are represented in the table 1, was used to model the milling dynamics. The cutter is considered as a system with two degrees of freedom. 
Table 1. The tool parameters

\begin{tabular}{|c|c|c|c|c|c|c|c|c|c|}
\hline $\begin{array}{c}d, \\
\mathrm{~mm}\end{array}$ & $n_{t}$ & $m_{1}, \mathrm{~kg}$ & $m_{2}, \mathrm{~kg}$ & $f_{1}, \mathrm{~Hz}$ & $f_{2}, \mathrm{~Hz}$ & $\xi_{1}$ & $\xi_{2}$ & $E, \mathrm{MPa}$ & $\mu$ \\
\hline 6 & 2 & 0.03993 & 0.03993 & 922 & 922 & 0.011 & 0.011 & $2 * 10^{\wedge} 5$ & 0.25 \\
\hline
\end{tabular}

where $d$ - the cutter diameter, $n_{t}$ - the number of teeth, $m_{1}, m_{2}$ - the modal masses, $f_{1}, f_{2}$ natural frequencies , $\xi_{1}, \xi_{2}$ - the modal dampings, $E$ - an elastic modulus, $\mu$ - Poisson's ratio. The machining mode parameters are given in the table 2 .

Table 2. The machining mode parameters

\begin{tabular}{|c|c|c|c|c|}
\hline $\begin{array}{c}\text { The } \\
\text { number of } \\
\text { calculation }\end{array}$ & $\begin{array}{c}\text { The spindle rotational } \\
\text { velocity, } \Omega \mathrm{rpm}\end{array}$ & $\begin{array}{c}\text { Feed, } s \\
\mathrm{~mm} / \text { tooth }\end{array}$ & $\begin{array}{c}\text { Radial depth of } \\
\text { cut, } w_{r} \mathrm{~mm}\end{array}$ & $\begin{array}{c}\text { Axial depth of } \\
\text { cut, } w_{a} \mathrm{~mm}\end{array}$ \\
\hline 1 & $5000: 200: 25000$ & 0.05 & 0.6 & 1 \\
\hline 2 & $5000: 200: 25000$ & 0.05 & 0.6 & 2 \\
\hline 3 & $5000: 200: 25000$ & 0.05 & 0.6 & 3 \\
\hline$\ldots$ & $\ldots$ & $\ldots$ & $\ldots$ & $\ldots$ \\
\hline 10 & $5000: 200: 25000$ & 0.05 & 0.6 & 10 \\
\hline
\end{tabular}

The linear model with the following coefficients $K_{t}=6^{*} 10^{\wedge} 8 \mathrm{~N} / \mathrm{m}^{\wedge} 2$ and $K_{a}=2 * 10^{\wedge} 8$ $\mathrm{N} / \mathrm{m}^{\wedge} 2$ (where $K_{t}$-tangential cutting coefficient, $K_{a}$ - axial cutting coefficient) [13] was used to calculate the cutting forces.

The tool acceleration signals for a certain mode (Fig.3) were received as a result of the multivariant modeling in the software package 3DCUT.
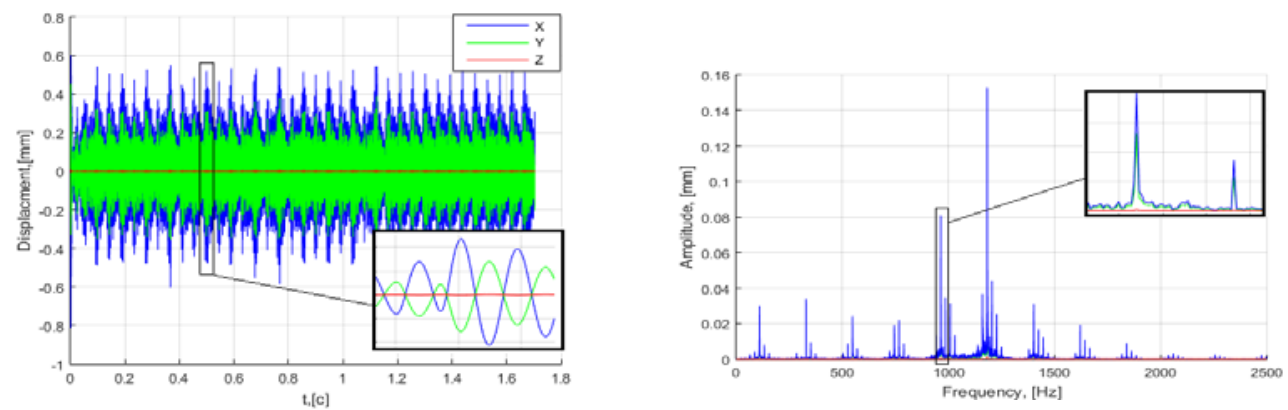

Fig. 3. The milling dynamics modeling results in the software package 3DCUT for the mode with the following parameters $\left(\Omega=6600 \mathrm{rpm}, s=0.05 \mathrm{~mm} /\right.$ tooth, $\left.w_{r}=0.6 \mathrm{~mm}, w_{a}=1 \mathrm{~mm}\right)$

The machining modes map (Fig. 4) was drawn as a result of the tool accelerarion signals processing with the detection algorithm.

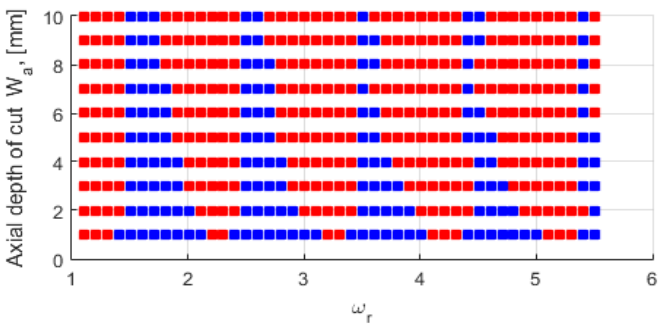

Fig. 4. The machining modes map. 
« The machining modes map » (Fig.4) is a diagram, whose x-axis is used to specify the unitless tool rotational frequency (6) and y-axis to specify the axial depth of cut $w_{a}$. Red color in the diagram represents the domains in which "chatter" takes place, whereas blue color represents the domains in which forced vibrations occur. There is almost the same representation of the detection results in the stability lobes diagram : the lobes, where the cutting mode is unstable, are drawn in the machining modes map (Fig.4).

$$
\omega_{r}=\frac{f_{1}}{\Omega n_{t}}
$$

As it can be seen in the machining modes map, the following parameters ensure the best efficiency: $\omega_{r}=1.6$ (the spindle rotational velocity $\Omega=17600 \mathrm{rpm}$ ), $w_{r}=0.6 \mathrm{~mm}, w_{a}=10$ $\mathrm{mm}$. The system tool/workpiece will vibrate at the frequency factor of the cutting edges with low amplitudes. This mode will allow to obtain the required quality of the machined surface and maintain high efficiency of the process.

\section{References}

1. Y. Altintas, Manufacturing automation: metal cutting mechanics, machine tool vibrations, and CNC design (Cambridge university press, 2000)

2. N. Van Dijk, Active chatter control in high-speed milling processes, (Dissertation, Eindhoven Univ. of Technology, 2011)

3. A. Gerasimenko, M. Guskov, A. Gouskov, P. Lorong, A. Shokhin, International Journal of Machining and Machinability of Materials, 19(1), pp. 17-40 (2017)

4. W. Gresik, Advanced machining processes of metallic materials: theory, modelling and applications (Elsevier, 2008)

5. Y. Altintas, Analytical prediction of three dimensional chatter stability in milling, JSME International Journal Series C Mechanical Systems, Machine Elements and Manufacturing, 44(3), pp. 717-723 (2001)

6. J. Tlusty, M. Polacek, The stability of machine tools against self-excited vibrations in machining, International Research in Production Engineering, pp. 465-474 (1963)

7. Gerasimenko A. et al., Variable compliance-related aspects of chatter in turning thinwalled tubular parts, Procedia CIRP, 31, pp. 58-63 (2015)

8. Kiselev I. A. et al., Three-dimensional modeling of deep hole vibratory drilling dynamics, Procedia Engineering, 176, pp. 50-55 (2017)

9. I. Kiselev, S. Voronov, Proceedings ASME 2014 International Design Engineering Technical Conferences and Computers and Information in Engineering Conference, p. V006T10A075 (2014)

10. N. Golyandina, E. Osipov, The «Caterpillar»-SSA method for analysis of time series with missing values (Journal of Statistical planning and Inference, 2007)

11. V. Kuts, S. Nikolaev, I. Ivanov, A new method for chatter detection in milling. Part 1 : Description and approbation, Scientific open access journal "Naukovedenie", v. 8(6) (2016)

12. I. Kiselev, S. Voronov, S. Arshinov, Proceedings ASME 2014 International Design Engineering Technical Conferences and Computers and Information in Engineering Conference, p. V006T10A076 (2014) 